\title{
Nutrición total en la alimentación del pollo de carne
}

\author{
Total nutrition in broiler feeding \\ 'Sergio Del Carpio H ${ }^{\text {a }}$, Pedro Del Carpio R a , 'Carolina Aguilar P'.
}

\section{RESUMEN}

El presente trabajo de investigación se realizó con el objetivo de reemplazar al antibiótico promotor del crecimiento en la dieta de pollos de carne por principios nutracéuticos sin atentar contra el rendimiento.

Se emplearon 300 pollos, de ambos sexos, en un ensayo de 7 semanas de duración en el que se evaluaron los siguientes tratamientos: T1 (Dieta tradicional sin antibiótico promotor del crecimiento), T2 (Dieta tradicional con antibiótico promotor del crecimiento), T3 (Dieta con nutracéuticos, sin antibiótico promotor del crecimiento) y T4 (Dieta con nutracéuticos, con antibiótico promotor del crecimiento). Cada pollito fue asignado aleatoriamente a cada uno de los tratamientos. Se prepararon raciones iso - energéticas e iso - proteicas para cubrir las necesidades nutritivas de las aves. El análisis estadístico se hizo a través de un Diseño Irrestrictamente al Azar con sub muestreo.

Los principios nutracéuticos sin antibiótico promotor del crecimiento propiciaron mayor consumo de alimento, mayores incrementos de peso y más eficiente utilización de los alimentos; pero mérito económico más alto, debido al encarecimiento de las raciones. En cuanto a eficiencia biológica las fuentes nutracéuticas evaluadas si pueden reemplazar al antibiótico promotor del crecimiento; siendo recomendable realizar la investigación pertinente para mejorar el mérito económico.

Palabras clave: Nutracéuticos, promotor del crecimiento, antibiótico - resistencia, pollos de carne.

\begin{abstract}
The present investigation was conducted with the aim of replacing the antibiotic growth promoter in broilers diet for nutraceutical principles without adversely affecting performance.

300 chickens were used, of both sexes, in a trial of seven weeks duration in which the following treatments were evaluated : T1 ( traditional diet without antibiotic growth promoter), T2 ( Traditional Diet antibiotic growth promoter ), T3 ( diet nutraceutical, without antibiotic growth promoter) and T4 ( diet with nutraceutical growth promoter antibiotic ) . Each chick was randomly assigned to each treatment. Rations were prepared iso - energetic and iso - protein to meet the nutritional needs of the birds. The statistical analysis was made through a design with sub unreservedly Random sampling.

The principles nutraceuticals without antibiotic growth promoter led to increased feed intake, weight gains greater and more efficient use of food, but higher economic merit due to higher rations. Regarding biological efficiency evaluated nutraceutical sources if they can replace antibiotic growth promoter, being recommended to perform relevant research to improve the economic merit.
\end{abstract}

\section{Key words:}

${ }^{1}$ Centro de Investigación Pecuaria. Facultad de Ingeniería Zootecnia. Universidad Nacional Pedro Ruiz Gallo. Lambayeque.
${ }^{a}$ Ingeniero Zootecnista 


\section{INTRODUCCIÓN}

La producción de alimentos, tanto de origen animal como vegetal, para consumo humano centra gran parte de su importancia en el hecho de que estas sean saludables; es decir, que bajo ningún punto de vista sean nocivos per se para las personas que los consumen.

- Los antibióticos promotores del crecimiento, además de las vacunas y la Zootecnia, han permitido que la producción avícola llegue hasta los niveles en que se encuentra. Sin embargo, se presenta la siguiente problemática:

- La tremenda mejora genética sólo ha sido sostenible a costa de hacer, cada vez más, menos inmunocompetentes a las aves. Los actuales pollos de carne, pavos de carne, gallina ponedora, etc. que producen a grandes niveles y velocidades, inimaginables hace menos de una década, son muy susceptibles al más pequeño desafío en salud por parte del ambiente. Esta es una de las razones por las que se emplearon sofisticadas gamas de antibactcrianos, entre los que se encuentran los promotores del crecimiento. Según muchos investigadores, en este momento la industria avícola se sustenta en animales tan ineptos en inmunocompetencia que si una bacteria mutara, haciéndose muy virulenta con rapidez, la industria podría desaparecer.

- Los antimicrobiales promotores del crecimiento han desafiado a las bacterias gastrointestinales a tal punto que han propiciado, según evidencias en Europa y Estados Unidos, la aparición de cepas bactcrianas resistentes a los antibióticos y que transmiten genéticamente esta habilidad a las bacterias que colonizan, normalmente, el tracto gastrointestinal humano; de tal manera que, frente a un desafío infeccioso, no habría respuesta a la terapia con antibióticos.

- Europa, Estados Unidos y Japón ya han prohibido el empleo de antibióticos promotores del crecimiento en aves y cerdos; tal prohibición debería hacerse, progresivamente, extensiva a los países del segundo y tercer mundo, debido a que las personas se merecen tal mejora; sin embargo, el rendimiento de los animales tiende a mermar. Por esta razón, muchos sectores de la industria de producción de alimentos de origen animal, avícola principalmente, podrían quebrar.

Por lo antes expuesto cabría plantear las siguientes preguntas:
¿Qué alternativas se podrían emplear para poder sustituirlos sin afectar el rendimiento animal?

¿Podrán los productos hasta ahora evaluados respaldar los niveles productivos como lo han hecho en otros países?

¿Existirán productos que sean nuevos aportantes de nutricinas?

Habiéndose planteado como hipótesis que el empleo simultáneo de varias fuentes de nutracéuticos, de diferentes modos de acción, en la dieta del pollo de carne permitirá la sustitución eficiente del antibiótico promotor del crecimiento sin afectar negativamente el rendimiento. Con el objetivo de lograr rendimiento similar al sustituir el antibiótico promotor del crecimiento por nutracéuticos.

\section{MATERIALYMÉTODOS}

\section{Localización y Duración}

El presente trabajo de investigación se desarrolló en la Unidad de Producción - Aves de la Facultad de Zootecnia, Universidad Nacional "Pedro Ruiz Gallo"; a $2 \mathrm{Km}$ hacia el oeste de la ciudad de Lambayeque. La fase experimental tuvo una duración de 7 semanas.

\section{Tratamientos Evaluados.}

T1: Dieta tradicional, sin empleo de farmacéutico y nutracéuticos.

T2:Dieta tradicional, con farmacéutico, sin nutracéuticos.

T3: Dieta con nutracéuticos, sin farmacéutico.

T4: Dieta con nutracéuticos y con farmacéutico.

\section{Características del Material y Equipo Experimentales.}

Se emplearon 300 pollos Cobb de un día de edad, de ambos sexos. Se emplearon raciones con 13.2 Mega julios (MJ) de Energía Metabolizable (EM) y 21\% de Proteína Cruda (PC) hasta los 21 días de edad; y 13.4 MJ de EM y $20 \%$ de PC entre los 22 y 49 días de edad. Los productos empleados para desarrollar el concepto de Nutrición Total fueron:

(1). Aviguard $\mathbb{R}$; (2). Leche Nido $\mathbb{R}$ crecimiento con Prebio 1; (3). Fndox $\mathbb{R} ;$ (4) Moldzap $\mathbb{R} ;(5)$.

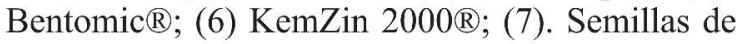
Achiote; (8). Semillas de Molle; (9). Allzyme Veg Pro ${ }^{\circledR} ;(10)$ Aceite de pescado acidulado desodorizado estabilizado; (11) Bicarbonato de sodio. Cuatro cercos para pollitos BB; una criadora de gas; corraletas de $5 \mathrm{~m}^{2}$; comederos de bandeja y de tolva; bebederos tipo sifón y lineales; balanzas, de precisión, tipo reloj y de plataforma; cintas plásticas y plumones de tinta indeleble; planillas para registro de información. 


\section{Descripción de la Metodología.}

Para contrastar las hipótesis se empleó un Diseño Completamente Azarizado con sub - muestreo, descrito por el siguiente modelo aditivo lineal: $\mathrm{Y}_{\mathrm{ijk}}=\mu+\tau_{\mathrm{i}}+\xi_{\mathrm{ij}}+\gamma_{\mathrm{ijk}}$

Se asumió una máxima probabilidad de $5 \%$ de cometer error de Tipo I.

Los pollitos se recibieron en cuatro cercos de crianza ( 75 por cerco), abastecidos por unla criadora de gas, bebederos tipo sifón y comederos de bandeja. Cada pollito fue identificado con una banda plástica numerada sujeta al tarso y pesado en balanza electrónica de precisión; luego las pesadas se realizaron cada 7 días hasta llegar a los 49 días de edad. La información fue anotada en planillas y almacenada en un cuaderno.

La criadora fue retirada a $\operatorname{los} 21$ días y a los 28 días se conformaron las sub - muestras, asignándolas a las corraletas (12 en total); aquí contaron con comederos tipo tolva y bebederos lineales.

El consumo de alimento fue determinado por diferencia entre la cantidad suministrada y el residuo, todos los días.

El producto Aviguard fue suministrado en el agua de bebida (hervida fría, sin proceso de potabilización) al momento de la recepción de los pollos. Siendo un producto aportante de microorganismos se pretendió, por competencia excluyente, evitar o minimizar la colonización del intestino por microorganismos nocivos.

La leche con inulina se incorporó a la dieta en la proporción de $1 \%$; además de aportar el prebiótico, la leche tiene la propiedad de acidificar el tracto propiciando la generación del medio adecuado para gérmenes benéficos; así como abastecer de ácido linoleíco conjugado que estimula la inmunocompetencia.

Los productos Endox, Moldzap y Bentomic fueron incorporados al maíz molido y arroz ñelén inmediatamente después de adquiridos, en las dosis mayores recomendadas por el fabricante; el resto de productos se incorporaron al momento de preparar la ración. Las semillas de Achiote y de Molle fueron molidas e incorporadas en la proporción de $0.5 \mathrm{y}$ $0.25 \mathrm{~kg}$, respectivamente, por cada $100 \mathrm{~kg}$ de peso vivo, como abastecedores de aceites esenciales. El complejo enzimático Allzyme Veg Pro (1 kg/TM) tuvo la finalidad de ayudar a las endoenzimas y complementarlas en su acción de degradación de principios de poca digestibilidad. El bicarbonato de sodio permite complementar el transporte de nutrientes y la digestión. El aceite de pescado acidulado desodorizado estabilizado se empleó como aportante de factores $\omega 3$.

El alimento fue preparado en loza de concreto y con palana; se tuvo especial cuidado en la homogeneización de la mezcla.

Las instalaciones fueron limpiadas y desinfectadas, así como el equipo y mantas, con una anticipación de 20 días. Una semana antes de la llegada de los pollos se hizo vacío sanitario, se extendió el material de cama y se aplicó formol. Todas las aves recibieron el programa de vacunación adecuado.

Se generó información para evaluar las siguientes variables:

- Consumo de alimento

- Peso y cambios en el peso corporal

- Conversión alimenticia

- Mérito económico

El análisis estadístico implicó la ejecución de pruebas de homogeneidad de varianzas; regresión lineal para estandarizar los incrementos de peso; dócima de F para determinar la significación de las diferencias entre tratamientos; análisis de covarianza entre peso inicial e incrementos de peso.

\section{RESULTADOS}

Los resultados referidos al Consumo de Alimento, Pesos e Incrementos de Peso, Conversión Alimenticia y Mérito Económico son mostrados en la Tabla $\mathrm{N}^{\circ} 01$.

El consumo de los tratamientos 1 y 3 fue superior al de los tratamientos 2 y 4 . El consumo total $(3.5 \mathrm{~kg})$ y diario ( $71 \mathrm{~g}$ ) fue similar en los tratamientos 1 y 3 ; en tanto que en los tratamientos 2 y 4 , aunque parecidos, en el tratamiento 2 el consumo fue ligeramente superior (4.2\%).

I a explicación al menor consumo de alimento en las dietas con presencia de antibiótico promotor del crecimiento se centraría en el efecto neto de su empleo; según MELLOR (2000) se gasta menos en alimento ingerido en el mantenimiento tanto del forro intestinal como de la superficie de absorción de nutrientes; se da un ahorro de glucosa, aminoácidos minerales, que habrían permanecido indisponibles para el organismo aviar.

Frente a los ahorros nutricionales reportados, el ave, en respuesta a una señal orgánica, reduciría el consumo de alimento. Sin embargo, tales ahorros no necesariamente se reflejarían en mayor rendimiento, ya que para ello se requeriría de factores estimulantes del anabolismo que permitan mayor síntesis de tejido (principalmente muscular). 
Tabla 1. Consumo de alimento, incrementos de peso, Conversión Alimenticia (C.A.) y Mérito Económico (M.E.) en pollos Cobb que recibieron nutracéuticos por farmacéuticos en la dieta.

\section{Tratamientos}

\section{Aspectos}

\begin{tabular}{lcccc} 
& $\mathbf{1}$ & $\mathbf{2}$ & $\mathbf{3}$ & $\mathbf{4}$ \\
\hline Duración experimental, semanas & $\mathbf{0 7}$ & $\mathbf{0 7}$ & $\mathbf{0 7}$ & $\mathbf{0 7}$ \\
Aves por tratamiento & $\mathbf{7 5}$ & $\mathbf{7 5}$ & $\mathbf{7 5}$ & $\mathbf{7 5}$ \\
Antibiótico en la dieta & No & Sí & No & Sí \\
Nutracéuticos en la dieta & $\mathbf{N o}$ & No & Sí & Sí \\
Consumo total/pollo, kg/período & $\mathbf{3 . 5 0 7}$ & $\mathbf{3 . 2 6 7}$ & $\mathbf{3 . 5 0 4}$ & $\mathbf{3 . 1 4 5}$ \\
Consumo diario/pollo, g/día & $\mathbf{7 1 . 6 0}$ & $\mathbf{6 6 . 9 0}$ & $\mathbf{7 1 . 5 1}$ & $\mathbf{4 . 2 0}$ \\
Incremento total peso, g/pollo & $\mathbf{2 1 8 2 . 8 ^ { \mathbf { a } }}$ & $\mathbf{1 9 4 9 . 6 ^ { \mathbf { b } }}$ & $\mathbf{2 2 0 3 . 5 ^ { \mathrm { a } }}$ & $\mathbf{1 8 8 4}^{\mathrm{c}}$ \\
Incremento diario, g/pollo & $\mathbf{4 4 . 5 5}$ & $\mathbf{3 9 . 7 9}$ & $\mathbf{4 4 . 9 7}$ & $\mathbf{3 8 . 4 5}$ \\
C.A. & $\mathbf{1 . 6 1}$ & $\mathbf{1 . 6 8}$ & $\mathbf{1 . 5 9}$ & $\mathbf{1 . 6 7}$ \\
M.E. & $\mathbf{1 . 3 4}$ & $\mathbf{1 . 4 4}$ & $\mathbf{1 . 6 1}$ & $\mathbf{1 . 7 3}$ \\
\hline
\end{tabular}

$\mathrm{a}, \mathrm{b}$

Letras diferentes sobre los promedios indican diferencias significativas entre tratamientos ( $\mathrm{P}=0.05$, Duncan $)$

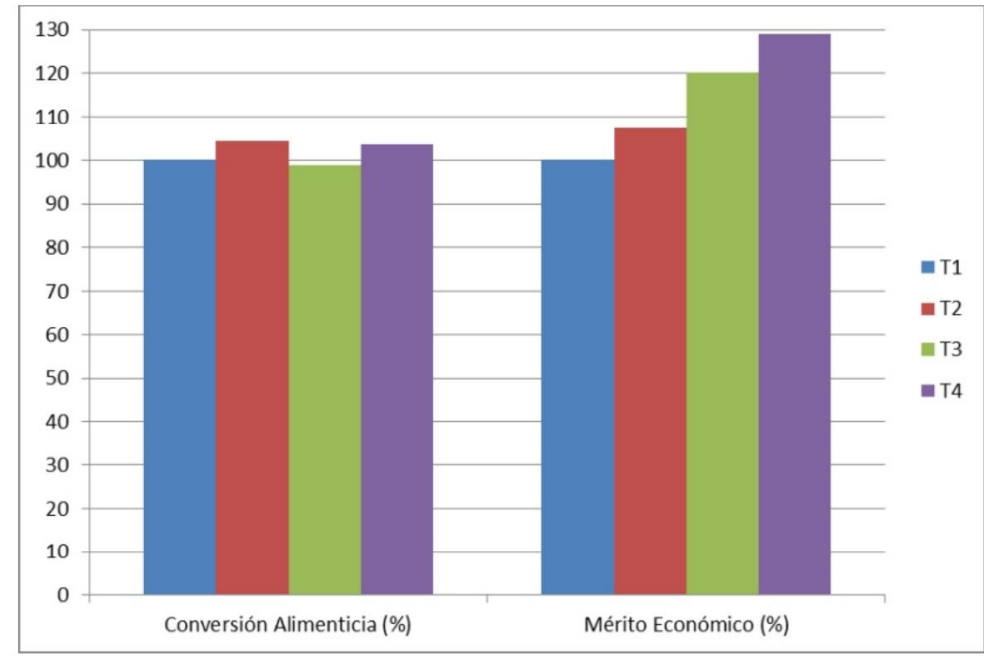

\begin{tabular}{|c|c|c|c|c|}
\hline 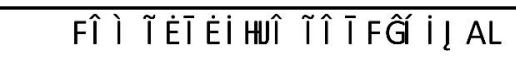 & $\overline{\mathrm{i} \hat{C}}$ & $\bar{i} \bar{C}$ & $\dot{i} \dot{C}$ & İ Ç \\
\hline 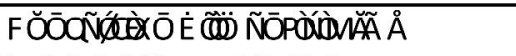 & ĈĆĆ & $\hat{C} C ́ C ̧ \not \dot{E}$ & EĐAD & ĈĆĆĊ \\
\hline 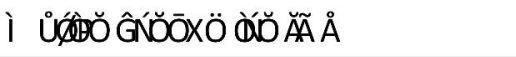 & Ĉćć & ĈĆĐA & ĈČć,1 & $\hat{C} C ̌ E / \hat{E}$ \\
\hline
\end{tabular}

Figura 1. Comparativo porcentual de Conversión alimenticia y Mérito económico en pollos Cobb que recibieron nutracéuticos por farmacéuticos en la dieta.

\section{DISCUSIÓN}

No se puede atribuir mayor o menor consumo a la concentración energética de las dietas, ya que estas fueron formuladas para ser muy parecidas en densidad energética y nivel proteico. Al respecto, NARAHARI (2000) y LEESON (2002), en función de los resultados de una serie de trabajos de investigación, son concluyentes al afirmar que la prioridad de las aves al consumir alimentos es abastecerse de energía y con una mayor densidad energética en la dieta los animales comerán menos, que no es el caso del presente ensayo. Los niveles de superioridad en el consumo (los tratamientos sin antibiótico superan en consumo total a los tratamientos con antibiótico entre 7 y $11 \%$ ) no pueden ser atribuidos completamente, tampoco, a efectos sobre la movilidad de la digesta a nivel intestinal. Por presencia de prebióticos (tipo inulina) en la dieta se produciría mayor concentración de ácidos grasos de cadena corta que ejercerían un efecto sobre la motilidad intestinal; se incrementa la frecuencia peristáltica, disminuye el tiempo de tránsito y se fomenta la disminución de sustrato del íleon (KAMATH et al., 1987; FICH et al., 1988), al desocuparse el intestino delgado se 
promovería un vaciado más rápido del estómago y se estimularía mayor consumo. Pero, el tratamiento 1 no poseía la fuente aportante de inulina $y$, contrario sensu, el tratamiento 4 poseía la fuente aportante de inulina pero también antibiótico promotor del crecimiento; por lo que la disminución del consumo es atribuible a la presencia del antibiótico promotor del crecimiento en la dieta (disminución de 7\% en el consumo total en T2) y a su interacción con los factores nutracéuticos evaluados (disminución de $11 \%$ en el consumo total en T4).

Como en el caso del consumo de alimentos, donde hubo antibióticos los incrementos de peso tendieron a ser menores. Dado que las ganancias de peso son reflejo de las cantidades de alimento consumidas, se pueden apreciar las mismas tendencias para el comportamiento de los tratamientos en ambos casos.

Es evidente que la mayor cantidad de alimento consumido propició que se absorba una mayor cantidad de nutrientes, lo que conllevó a una mayor cantidad de tejido sintetizado. Se esperaba, a priori, que el tratamiento 1 ( $\sin$ antibiótico y sin nutracéuticos) fuese el más afectado negativamente por las circunstancias adversas que se dan en toda crianza intensiva (diez mil pollos por galpón); sin embargo, en un trabajo de investigación no necesariamente ocurre eso, aún más si las instalaciones estuvieron descansadas y fueron correctamente limpiadas y desinfectadas.

Como en el caso del consumo, se asume que el efecto sobre la microbiota colónica tuvo un marcado efecto sobre los resultados logrados con los incrementos de peso. Al controlarse el desafío sanitario (normal en toda explotación avícola y que se incrementa con la edad) no hubo una proliferación de bacterias del tipo Clostridium y Escherichia, entre otras consideradas nocivas; permitiendo una microbiota equilibrada o en la que predominaran bacterias benéficas favoreciendo la absorción de nutrientes y los incrementos de peso.

Al comparar las conversiones alimenticias (C.A.) se aprecia que los tratamientos 1,3 y 4 lograron 95.8 , 94.6 y $99.4 \%$, respectivamente, con relación a la C.A. lograda por el tratamiento 2. En función de estos resultados se puede inferir que en el tratamiento 3 no sólo hubo una mayor disponibilidad de nutrientes a nivel del intestino, sino que también una mayor tasa de absorción y fueron empleados más eficientemente en las funciones de síntesis de tejido. La observación de las C.A. generales muestra que las logradas con los tratamientos que incluyeron antibiótico promotor del crecimiento no fueron malas, sino todo lo contrario, pero los valores logrados con el tratamiento 3 fueron superiores.

Como se indicó anteriormente, el empleo de diferentes productos sin antibiótico promotor del crecimiento en la dieta puede permitir resultados similares o mejores que con la presencia de antibiótico (DAMME, 1999; MELLOR, 2000; LANGHOUT, 2000; MANDAL et al., 2000; ATHER, 2000; WILLIAMS y LOSA, 2001).

Debería determinarse si es conveniente el empleo de fuentes aportantes de aceites esenciales en conjunto con un suplemento de exo - enzimas, ya que no se ha determinado si tendrían un efecto sinérgico o antagónico.

Los valores de mérito económico (M.E.) fueron $1.34,1.44,1.61$ y 1.73 respectivamente para los tratamientos $1,2,3$ y 4 . Si el tratamiento 2 , que incluyó antibiótico promotor del crecimiento en la dieta, es empleado como el tratamiento referencial entonces los M.E. de los tratamientos 1,3 y 4 representan $93.1,111.81$ y $120.14 \%$, respectivamente. Por lo tanto, en el tratamiento 3 (sin antibiótico promotor del crecimiento y con nutracéuticos en la dieta) se gastó $11.8 \%$ más en alimento para ganar $1 \mathrm{~kg}$ de peso en comparación con el tratamiento 2. Las ventajas de $13 \%$ en incremento de peso y de $5.4 \%$ en C.A. del tratamiento 3 sobre el tratamiento 2 no fue suficiente, en el presente ensayo, para compensar los incrementos en costo por kg de ración. El empleo de diferentes productos para tratar de lograr los principios de Nutrición Total encareció a la ración de inicio en $27 \%$ y a la ración de crecimiento en $16 \%$ en comparación con el tratamiento 2. Por supuesto que se puede optar por una serie de estrategias para minimizar este impacto sobre el costo de las raciones.

Una puede ser el empleo de cereales alternativos de menor costo que el maíz; contando con el aporte de exo - enzimas y considerando la estimulación en la secreción de endoenzimas por parte de los aceites esenciales contenidos en especias y hierbas, varios cereales pueden reemplazar casi totalmente al maíz, el que sufre grandes variaciones en el precio. Otra alternativa puede consistir en determinar si todos los productos evaluados tienen efectos sinérgicos o antagónicos; aunque evaluados aisladamente han presentado efectos benéficos, actuando en conjunto su comportamiento puede neutralizarse o ser negativo; la determinación de esto podría significar el retiro de uno o más de la fórmula, abaratándola sin perder eficiencia. Así mismo, conforme ha transcurrido el tiempo se dispone de nuevas fuentes de prebióticos; la inulina ha mostrado su bondad, aún cuando la fuente empleada haya sido alimento para mascotas (LUJAN, 2002), se consideró que la 
leche de empleo humano, de hecho, es una fuente más adecuada; pero en este momento se dispone de oligosacáridos mananos que también tienen efecto prebiótico $\mathrm{y}$, aunque su costo por $\mathrm{kg}$ puede ser mayor, se emplean a niveles de hasta la décima parte del empleado en el presente ensayo la leche con inulina. Con todo, cabría preguntarse si los consumidores estarían o no dispuestos a pagar un ligero plus por carne más segura.

\section{CONCLUSIONES}

La presencia de antibiótico promotor del crecimiento en la dieta tendió a disminuir el consumo de alimento, entre 7 y $11 \%$ en comparación al tratamiento en que se emplearon principios nutracéuticos.

E1 empleo de principios nutracéuticos, sin antibiótico promotor del crecimiento, permitió mayores incrementos de peso que en los tratamientos en los que se empleó antibiótico promotor del crecimiento; en magnitudes entre 13 y $17 \%$.

La eficiencia de utilización de los alimentos fue mejor cuando se emplearon insumos aportantes de nutracéuticos sin utilizar antibiótico promotor del crecimiento; la ventaja en conversión alimenticia fue del orden de $5 \%$.

El empleo de insumos aportantes de nutracéuticos encareció las raciones, motivo por el cual el mérito económico de la ración con nutracéuticos, sin antibiótico, fue $12 \%$ menos eficiente que el de la ración que no empleó nutracéuticos, pero si antibiótico promotor del crecimiento.

\section{AGRADECIMIENTOS}

A Dios, a mi familia y a las personas que colaboraron en la realización del presente trabajo.

\section{REFERENCIAS BIBLIOGRAFICAS}

Ather, M. 2000. Polyherbal additive proves effective against vertical transmission of IRD. World Poultry-Elsevier, 16(11): 50-51.

Damme, K. 1999. Natural enhancers could replace antibiotics in turkey feed. World Poultry-Elsevier, 15(09): 27-28.

Fich, A.; S.F. Phillips; N.S. Hakimb; M.L. Brown, and A.R. Zinsmeister. 1989. Stimulation of ileal emptying by short-chain fatty acids. Dig. Dis. Sci., 34:1516.

Kamath, P.S.; M.T. Hoepfner, and S.F. Phillips. 1987. Short-chain fatty acids stimulate motility of the canine ileum. Am. J. Physiolog., 253: G427.

Langhout, P. 2000. New additives for broiler chickens. World Poultry-Elsevier, 16(03): 2227.

Leeson, S. 2002. What motivates chickens to eat specific nutrients? World Poultry-Elsevier, 18(03): 15-16.

Lujan, E. 2002. Sustitución del antibiótico promotor del crecimiento por fuentes de pre- y pro-biótico en las dietas de pavos hybrid y su efecto sobre el rendimiento. Tesis. Facultad de Zootecnia, Universidad Nacional "Pedro Ruiz Gallo". Lambayeque, Perú.

Mandal, L.; T. Biswas, and S.K. Sarkar. 2000. Broilers perform well on herbs or enzymes in maize diets. World Poultry-Elsevier, 16(05): 19-21.

Mellor, S. 2000. Nutraceuticals - alternatives to antibiotics. World Poultry-Elsevier, 16(02): 30-33.

Narahari, D. 2000. Nutritional methods of improving feed efficiency. World PoultryFlsevier, 16(12): 16-19.

Schwarzer, K. and C.A. Adams. 2001. Oxidative stress and animal performance. Worldwide AGP Seminar. Kemin Lab. USA.

Williams, P. and R. LOSA. 2001. The use of essential oils and their compounds in poultry nutrition. World Poultry-Elsevier, 17(04): 1415.

\section{Correspondencia.}

Sergio Del Carpio Hernández.

Av. El Dorado 1331. Urb. Francisco Bolognesi. José Leonardo Ortiz. Chiclayo. sergiodelcarpio@hotmail.com 